\title{
Repackaging of Malfunctioning High-flow Nasal Cannula as a Rescue Oxygen Therapy: An Innovation amid COVID-19 Crisis
}

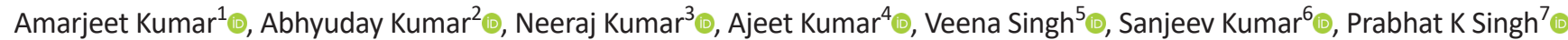

\begin{abstract}
Oxygen supplementation is required for approximately $14 \%$ of the patients diagnosed of having COVID-19 infection. Despite the use of conventional oxygen therapy, 5\% among these require treatment in the intensive care unit (ICU). Here, we are describing a situation in which oxygen therapy was delivered to the patients by making an assembly of oxygen tubing, three-way stopcock, and high-flow nasal cannula (HFNC) present in the hospital setting following the malfunction of air blender of HFNC machine (Fig. 1). This assembly might be useful as rescue oxygen therapy during a malfunction of HFNC machine and in resource-limited settings where HFNC machine is not available. The mechanisms of action could be (1) washout of anatomic dead space due to medium oxygen flow, (2) improved gas mixing in large airways, and (3) increased oxygen concentration inside the conducting airway.

Keywords: Acute hypoxic respiratory failure, COVID-19, High-flow nasal cannula.

Indian Journal of Critical Care Medicine (2021): 10.5005/jp-journals-10071-23953
\end{abstract}

\section{INTRODUCTION}

Oxygen supplementation is required for approximately $14 \%$ of the patients diagnosed of having COVID-19 infection. Despite the use of conventional oxygen therapy, $5 \%$ among these require treatment in the intensive care unit (ICU). Type 1 respiratory failure in these patients is due to an increased physiological dead space and increased oxygen demand due to high catabolic state as seen in non-COVID-19 acute respiratory distress syndrome (ARDS) patients. ${ }^{2}$ The Surviving Sepsis Campaign (SSC) guidelines for COVID-19 have suggested the benefit of a HFNC device over noninvasive ventilation (NIV) to obtain oxygen saturation $\left(\mathrm{SpO}_{2}\right)$ above $90 \%{ }^{3}$ The HFNC oxygen therapy allows warm and humidified oxygen with a maximum flow of $60 \mathrm{~L} /$ minute and positive end-expiratory pressure of up to $5 \mathrm{~cm}$ of $\mathrm{H}_{2} \mathrm{O}$.

Here, we are describing a scenario where oxygen therapy was delivered to the patients by making an assembly of oxygen tubing, three-way stopcock, and high-flow nasal cannula (HFNC) available in the hospital setting following the malfunction of air blender of HFNC machine (SAANS, InnAccel Technologies Pvt Ltd., Bengaluru, India). The two flowmeters were connected to a HFNC by using a three-way stopcock (Poly Medicure Ltd., Haryana, India) assembly as shown in Figure 1. A three-way stopcock may tolerate pressure up to $4.5 \mathrm{bar}$ ( $65 \mathrm{psi}$ ) and 360 rotations, and it consists of one rotating male luer and two female luer connectors. ${ }^{4}$ In this assembly, we connected one female luer of a three-way stopcock with oxygen tubing of one flowmeter, one male luer with another flowmeter, and a second female luer was connected with HFNC as shown in Figure 1.

\section{Case Description}

A 62-year-old COVID-19-positive female patient was admitted to our ICU with complaints of ARDS. Chest X-ray showed bilateral lung field opacities. On the 3rd day of admission to ICU, she was maintaining oxygenation on HFNC therapy with a flow of $30 \mathrm{~L} /$ minutes and $\mathrm{FiO}_{2}$ of $70 \%$. Unfortunately, the oxygen blender of the

\footnotetext{
1,3 Department of Trauma and Emergency, AllMS, Patna, Bihar, India

${ }^{2,4}$ Department of Anaesthesiology, AlIMS, Patna, Bihar, India

${ }^{5}$ Department of Burns and Plastic Surgery, AllMS, Patna, Bihar, India

${ }^{6}$ Department of CTVS, AlIMS, Patna, Bihar, India

${ }^{7}$ AllMS, Patna, Bihar, India
}

Corresponding Author: Amarjeet Kumar, Department of Trauma and Emergency, AlIMS, Patna, Bihar, India, Phone: +91 09570890646, e-mail: amarjeetdmch@gmail.com

How to cite this article: Kumar A, Kumar A, Kumar N, Kumar A, Singh V, Kumar $S$, et al. Repackaging of Malfunctioning High-flow Nasal Cannula as a Rescue Oxygen Therapy: An Innovation amid COVID-19 Crisis. Indian J Crit Care Med 2021;25(8):948-949.

Source of support: Nil

Conflict of interest: None

HFNC machine became malfunctional and oxygen desaturation started in the patient. She was having tachypnea (respiratory rate $>35$ ) and $\mathrm{SpO}_{2}$ fell to less than $90 \%$. We disconnected the nasal cannula from the HFNC machine and connected it with an oxygen flowmeter as shown in Figure 1. Soon, we detected $\mathrm{SpO}_{2}>95 \%$. The patient recovered successfully from respiratory distress.

\section{Discussion}

Spontaneously breathing patients with acute hypoxemic respiratory failure (AHRF) usually have a high respiratory drive with high minute ventilation, and thus a potentially damaging transpulmonary pressure variation. ${ }^{5}$ Noninvasive respiratory support is advised in COVID-19 respiratory failure patients for acute-on-chronic type II respiratory failure and as a bridge awaiting endotracheal intubation. The starting oxygen therapy of AHRF is via nasal cannula, face mask, non-rebreathing oxygen mask, and HFNC. As per SSC guidelines 


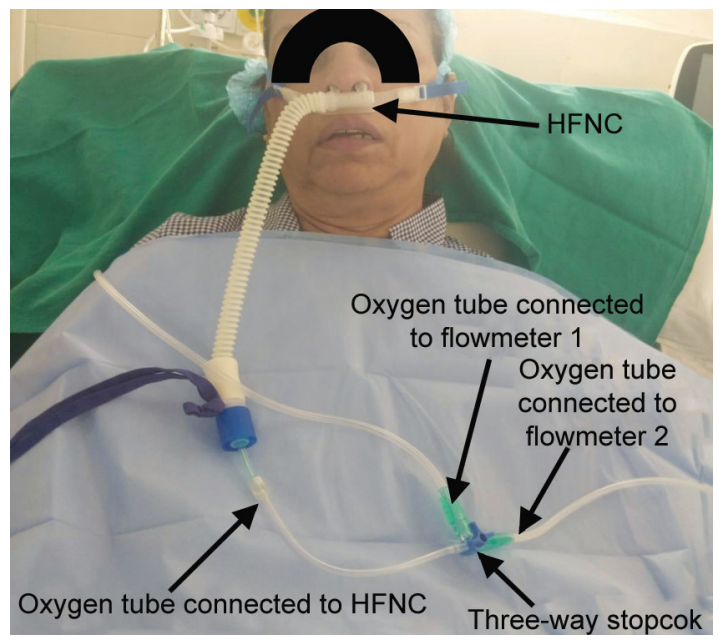

Fig. 1: High-flow nasal cannula-Three-way stopcock assembly

for COVID-19, HFNC is preferred over NIV. ${ }^{3}$ Recent studies in COVID-19 pandemic also observed the positive response of HFNC in moderate hypoxemic patients. ${ }^{6}$ We noticed an improvement in oxygen saturation after initial desaturation. By making the above assembly of oxygen tubing with a three-way stopcock and HFNC, we provided sufficient oxygenation to our patient following the malfunction of the HFNC machine. In our assembly, we used two oxygen flowmeters in the place of an oxygen blender as a source of oxygen to the HFNC. This assembly might be useful as rescue oxygen therapy in case of failure of a HFNC machine and in resourcelimited settings where HFNC machine is not available. This assembly provides medium oxygen flow (flow above the maximum capacity of a single flowmeter) to the nasal cannula without air blending. We confirmed the positive effect of two oxygen flowmeters over one flowmeter, as the closure of one flowmeter lead to a significant decrease in oxygenation parameters as measured by the $\mathrm{SpO}_{2}$. The physiology behind the clinical application of this assembly could be (1) washout of anatomic dead space due to medium oxygen flow, (2) improved gas mixing in large airways, and (3) increased oxygen concentration inside the conducting airway.

Interestingly, our patient did not complain of discomfort by using oxygen therapy using this assembly. This assembly is lightweight, easy to make, and cost-effective. This method is particularly helpful in resource-limited conditions like the current
COVID-19 pandemic to provide medium nasal cannula flow in a remote location with help of an oxygen cylinder.

Drawbacks: The high flow from the assembly can cause dryness of nasal mucosa and nasal bleeding and should be taken into account. Disconnection at three-way stopcock assembly might be a problem.

Dual oxygen flowmeter can be used to provide medium gas flow through a HFNC during the malfunction of the machine. Our conclusion is based on a single patient; hence, validation requires the volumetric calculation of actual oxygen flow at the patient end.

\section{OrCID}

Amarjeet Kumar (1) https://orcid.org/0000-0002-4272-5750

Abhyuday Kumar (1) https://orcid.org/0000-0002-9247-6713

Neeraj Kumar $\odot$ https://orcid.org/0000-0002-9161-7000

Ajeet Kumar $\odot$ https://orcid.org/0000-0002-1464-6684

Veena Singh (1) https://orcid.org/0000-0002-2120-6548

Sanjeev Kumar (1) https://orcid.org/0000-0003-4867-9326

Prabhat K Singh @ https://orcid.org/0000-0003-1888-4679

\section{References}

1. Wu Z, McGoogan JM. Characteristics of and important lessons from the coronavirus disease 2019 (COVID-19) outbreak in China: summary of a report of 72314 cases from the Chinese Center for Disease Control and Prevention. JAMA 2020;323(13):1239-1242. DOI: 10.1001/ jama.2020.2648.

2. Liu X, Liu X, Xu Y, Xu Z, Huang Y, Chen S, et al. Ventilatory ratio in hypercapnic mechanically ventilated patients with COVID-19 associated ARDS. Am J Respir Crit Care Med 2020;201(10):1297-1299. DOI: 10.1164/rccm.202002-0373LE.

3. Alhazzani W, Møller MH, Arabi YM, Loeb M, Gong MN, Fan E, et al. Surviving sepsis campaign: guidelines on the management of critically ill adults with coronavirus disease 2019 (COVID-19). Crit Care Med 2020. DOI: 10.1007/s00134-020-06022-5.

4. Availablefrom:http://www.polymedicure.com/?wpccategories=threeway-stop-cocks [Last accessed on Nov 28, 2020].

5. Gattinoni L, Coppola S, Cressoni M, Busana M, Rossi S, Chiumello D. COVID-19 does not lead to a "typical" acute respiratory distress syndrome. Am J Respir Crit Care Med 2020;201(10):1299-1300. DOI: 10.1164/rccm.202003-0817LE.

6. Wang K, Zhao W, Li J, Shu W, Duan J. The experience of high-flow nasal cannula in hospitalized patients with 2019 novel coronavirus-infected pneumonia in two hospitals of Chongqing, China. Ann Intensive Care 2020;10(1):37. DOI: 10.1186/s13613-020-00653-z. 